\title{
Search for the Standard Model Higgs Boson in the $\mathrm{H}$ to $\tau^{+} \tau^{-}$to lepton-hadron and hadron-hadron Decay Modes with the ATLAS Detector
}

\author{
Yuki Sakurai on behalf of the ATLAS Collaboration. \\ Waseda Research Institute for Science and Engineering, The Waseda University, Tokyo, Japan \\ E-mail: yuki.sakurai@cern.ch
}

\begin{abstract}
In this proceeding, a search for a Higgs boson in the $\mathrm{H}$ to $\tau^{+} \tau^{-}$decay mode, where both taus decay hadronically or one decay hadronically and one leptonically. The analysis is based on a data sample of proton-proton collision collected by the ATLAS detector at the Large Hadron Collider (LHC) at centre-of-mass energies of $7 \mathrm{TeV}$ during 2011, corresponding to an integrated luminosity of $4.7 \mathrm{fb}^{-1}$. No significant excess is observed in the Higgs boson mass range of $100-150 \mathrm{GeV}$. The observed (expected) limits on the cross-section times branching ratio for $\mathrm{H}$ to $\tau^{+} \tau^{-}$ at $m_{H}=125 \mathrm{GeV}$ are 6.2(5.9) and 6.3(6.5) for lepton-hadron and hadron-hadron decay modes, respectively.
\end{abstract}

Keywords: LHC, ATLAS, Higgs, tau

\section{Introduction}

The Higgs boson is a fundamental particle in the Standard Model (SM) of particle physics predicted by the Higgs mechanism, which is related to the mass generation mechanism. The $\mathrm{H} \rightarrow \tau^{+} \tau^{-}$decay mode is one of the most relevant search channels in the SM Higgs low-mass region. If the Higgs boson is independently discovered in this channel, the measurement of the $\mathrm{H} \rightarrow \tau^{+} \tau^{-}$decay rate provides a test of the SM prediction for the $\tau$ Yukawa coupling. In this proceeding, a search for the SM Higgs boson in $\mathrm{H} \rightarrow \tau_{\text {lep }} \tau_{\text {had }}$ and $\mathrm{H}$ $\rightarrow \tau_{\text {had }} \tau_{\text {had }}$ decay modes is reported, where $\tau_{\text {lep }}$ and $\tau_{\text {had }}$ denote leptonically and hadronically decaying $\tau$ lepton, respectively.

\section{The $\mathbf{H} \rightarrow \tau_{l e p} \tau_{\text {had }}$ search}

\subsection{Analysis selection and categories}

In this channel, a data sample collected with a single lepton trigger is analyzed, where the electron $E_{T}$ and muon $p_{T}$ thresholds are from $20 \mathrm{GeV}$ to $22 \mathrm{GeV}$ and 18 $\mathrm{GeV}$, respectively. Signal events are characterized by exactly one electron with $E_{T}>25 \mathrm{GeV}$ or one muon $p_{T}$
$>20 \mathrm{GeV}$, and one $\tau_{\text {had }}$ candidate with $p_{T}>20 \mathrm{GeV}$, imposing opposite charge between lepton and $\tau_{\text {had }}$ candidate. The transverse mass is expressed as

$$
m_{T}=\sqrt{2 p_{T}^{e / \mu} E_{T}^{m i s s}(1-\cos \Delta \phi)},
$$

where $\Delta \phi$ is the transverse angle between lepton and $E_{T}^{\text {miss }}$. In order to suppress $\mathrm{W}+$ jets background, events are selected with $m_{T}<30 \mathrm{GeV}$. After applying above selections, events are divided into several categories. The H+2jet Vector Boson Fusion (VBF) category is defined to enhance signal events from VBF process, which the VBF topology has two high-energy jets with a large rapidity separation in the final state. In this category, events include all selected events with $E_{T}^{\text {miss }}>20 \mathrm{GeV}$ and at least two jets with $p_{T}>25 \mathrm{GeV}$, imposing several VBF topological selections [2]. The $\mathrm{H}+1$ jet category include all selected events with $E_{T}^{\text {miss }}>20 \mathrm{GeV}$ and at least one jet with $p_{T}>25 \mathrm{GeV}$, that fail the VBF selection. The $\mathrm{H}+0 \mathrm{jet}$ category include all selected events without any jet with $p_{T}>25 \mathrm{GeV}$, and The analysis is separated into events with $E_{T}^{\text {miss }}>20 \mathrm{GeV}$ and $E_{T}^{\text {miss }}<20 \mathrm{GeV}$. The $\mathrm{e} \tau_{\text {had }}$ and $\mu \tau_{\text {had }}$ final states are separated in $\mathrm{H}+1$ jet and $\mathrm{H}+0$ jet categories. The Miss- 
ing Mass Calculator (MMC) is used for the Higgs mass reconstruction [4], and Figure 1 (left) shows the MMC mass distribution.

\subsection{Background estimation and modeling}

In order to estimate background contribution to $H \rightarrow$ $\tau_{\text {lep }} \tau_{\text {had }}$ signal, a data control sample referred as samesign (SS) sample is defined. It is applied to all event selections described in section 2.1, but requiring same charge between lepton and $\tau_{\text {had }}$. On the other hand, the signal sample is referred as opposite-sign (OS) sample. The number of OS background events in the signal region $\left(n_{O S}^{b k g}\right)$ can be expressed as

$$
n_{O S}^{\text {all }}=n_{S S}^{\text {all }}+n_{O S-S S}^{W+\text { jets }}+n_{O S-S S}^{Z \rightarrow \tau \tau}+n_{O S-S S}^{\text {other }},
$$

where $n_{S S}^{\text {all }}$ is all SS background and the remaining terms are the differences between the number of OS and SS events for $\mathrm{W}+$ jets, $Z / \gamma^{*} \rightarrow \tau \tau$ and other backgrounds, respectively. The multi-jet background is estimated directly from data SS events because of no charge correlation between two jets faking to lepton and $\tau_{\text {had }}$. For the $\mathrm{W}+$ jets background, there is a significant difference between the number of OS and SS events. Therefore, the $n_{O S-S S}^{W+j e t s}$ is estimated from the simulation after applying normalization factors from $\mathrm{W}$-dominated data control region.

\section{The $\mathbf{H} \rightarrow \tau_{\text {had }} \tau_{\text {had }}$ search}

\subsection{Analysis selection and categories}

The event selection starts with a double hadronic $\tau$ trigger, where the $p_{T}$ thresholds are $29 \mathrm{GeV}$ and 20 GeV. Signal events are characterized by two identified $\tau_{\text {had }}$ candidates with $p_{T}>35 \mathrm{GeV}$ and $p_{T}>25$ $\mathrm{GeV}$ selected with opposite charge. In this channel, only $\mathrm{H}+1$ jet category is defined. After selecting two $\tau_{\text {had }}$ candidates, the collinear mass approximation cuts $\left(0<x_{1}, x_{2}<1\right.$ and $\left.\Delta R(\tau, \tau)<2.2\right)$ are applied, where $x_{1}$ and $x_{2}$ are the momentum fractions of the two $\tau_{\text {had }}$ candidates carried away by their visible decay products. Then, $E_{T}^{m i s s}>20 \mathrm{GeV}$ and the leading jet with $p_{T}>$ $40 \mathrm{GeV}$ are required, imposing by $m_{\tau \tau j}>225 \mathrm{GeV}$. The collinear mass approximation is used for the Higgs mass reconstruction [4], and Figure 1 (right) shows the collinear mass distribution.

\subsection{Background estimation and modeling}

$Z / \gamma^{*} \rightarrow \tau \tau$ and multi-jet events contribute the main backgrounds to signal, and they are modeled by a datadriven method referred as the two-dimensional track multiplicity fit technique. These backgrounds are estimated by looking at tracks associated to the two $\tau_{\text {had }}$ candidates simultaneously, where tracks are counted in the cone defined by $\Delta R<0.6$. This technique is performed to a data-driven control region to obtain normalization of $Z / \gamma^{*} \rightarrow \tau \tau$ background. The multi-jet contribution is estimated by the same technique, where the fit is performed in the signal region. In this fit, the multi-jet template is modelled by data SS events while the $Z / \gamma^{*} \rightarrow \tau \tau$ is modelled by the simulation.
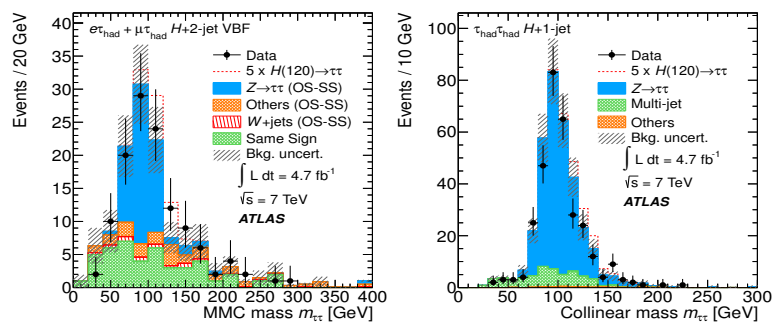

Figure 1: MMC mass distribution in $\mathrm{H} \rightarrow \tau_{\text {lep }} \tau_{\text {had }}$ channel for $\mathrm{H}+2 \mathrm{jets}$ (VBF) category (left), and collinear mass distribution in $\mathrm{H} \rightarrow \tau_{\text {had }} \tau_{\text {had }}$ channel for $\mathrm{H}+1$ jet category. Predictions from the Higgs boson signal $\left(m_{H}=120 \mathrm{GeV}\right)$ and from backgrounds are given. [2]

\section{Results}

No significant excess is observed in the data compared to the SM expectations in both channels. Therefore, exclusion limits at the $95 \%$ confidence level, normalized to the SM cross section times the branching ratio of $\mathrm{H} \rightarrow \tau \tau$, are extracted as a function of the Higgs boson mass. The observed (expected) limits at $m_{H}=$ $125 \mathrm{GeV}$ are 6.2(5.9) and 6.3(6.5) for $\mathrm{H} \rightarrow \tau_{\text {lep }} \tau_{\text {had }}$ and $\mathrm{H} \rightarrow \tau_{\text {had }} \tau_{\text {had }}$ channel, respectively. Figure 2 shows expected and observed limits for both channels.
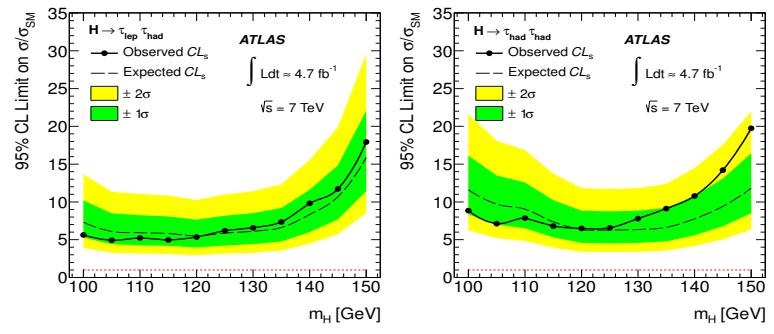

Figure 2: Observed and expected 95\% confidence level upper limits on the Higgs boson cross section times branching ratio, normalized to the Standard Model expectation, as a function of the Higgs boson mass for $\mathrm{H} \rightarrow \tau_{\text {lep }} \tau_{\text {had }}$ (left) and for $\mathrm{H} \rightarrow \tau_{\text {had }} \tau_{\text {had }}$ (right) channels. [2]

\section{References}

[1] ATLAS Collaboration, JINST 3 (2008) S08003

[2] ATLAS Collaboration, JHEP 09 (2012) 070, arXiv:1206.5971

[3] ATLAS Collaboration, ATLAS-CONF-2012-014, http://cdsweb.cern.ch/record/1429662

[4] A. Elton, P. Murat, A. Pranko and A. Safeness's, Nucl. Instrum. Meth. A 654 (2011) 481, arXiv:1012.4686 